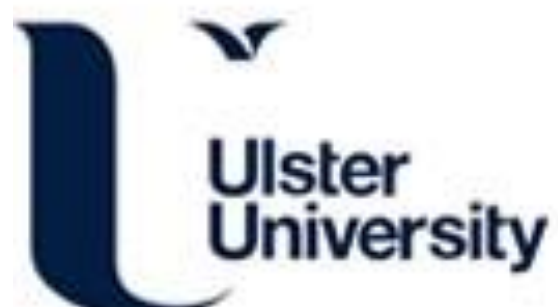

Raman study of multiwalled carbon nanotubes functionalized with oxygen groups

Murphy, H., Papakonstantinou, P., \& Okpalugo, TIT. (2006). Raman study of multiwalled carbon nanotubes functionalized with oxygen groups. JOURNAL OF VACUUM SCIENCE \& TECHNOLOGY B, 24(2), 715-720. https://doi.org/10.1116/1.2180257

Link to publication record in Ulster University Research Portal

\section{Published in:}

JOURNAL OF VACUUM SCIENCE \& TECHNOLOGY B

Publication Status:

Published (in print/issue): 01/03/2006

DOI:

10.1116/1.2180257

Document Version

Publisher's PDF, also known as Version of record

\section{General rights}

Copyright for the publications made accessible via Ulster University's Research Portal is retained by the author(s) and / or other copyright owners and it is a condition of accessing these publications that users recognise and abide by the legal requirements associated with these rights.

\section{Take down policy}

The Research Portal is Ulster University's institutional repository that provides access to Ulster's research outputs. Every effort has been made to ensure that content in the Research Portal does not infringe any person's rights, or applicable UK laws. If you discover content in the Research Portal that you believe breaches copyright or violates any law, please contact pure-support@ulster.ac.uk. 


\title{
Raman study of multiwalled carbon nanotubes functionalized with oxygen groups
}

\author{
H. Murphy, P. Papakonstantinou, ${ }^{\text {a) }}$ and T. I. T Okpalugo \\ Nanotechnology Research Institute, University of Ulster, Shore Road, BT37 OQB Northern Ireland, \\ United Kingdom
}

(Received 27 April 2005; accepted 30 January 2006; published 9 March 2006)

\begin{abstract}
We present changes in the first and second order Raman spectra of multiwalled carbon nanotubes (MWNTs) functionalized with oxygenated groups. The oxygen groups were introduced onto the nanotube surface through two strong acid purification routes: (1) reflux in concentrated $(70 \%)$ $\mathrm{HNO}_{3}$ acid for $4 \mathrm{~h}$ at $80{ }^{\circ} \mathrm{C}$ and (2) ultrasonification in $3 \mathrm{HNO}_{3}(70 \%): 1 \mathrm{H}_{2} \mathrm{SO}_{4}(98 \%)$ for $8.5 \mathrm{~h}$. Raman spectroscopy, using two laser excitation wavelengths (514.5 and $632.8 \mathrm{~nm}$ ), x-ray photoelectron spectroscopy, and thermal gravimetric analysis were employed to study the evolution of the products. All the techniques revealed a higher degree of functionalization for scheme 2 compared to scheme 1 . Charge transfer phenomena were manifested by a shift of the $\mathrm{C} 1 s$ core level towards higher binding energies. We found that the intensity of both the $D$ and $G$ energy Raman modes if normalized to the second order mode $D^{*}$ mode follows similar trends upon acid treatments. We interpret this result together with the observed dispersion of $G$ mode as an indication that the $G$ mode in carbon nanotubes is defect induced in a double resonant process. Both acid schemes cause an upshift of $D$ and $G$ Raman modes, due to intercalation of acid molecules, exerting pressure on the $s p^{2}$ structure and an electron transfer from the $\pi$ states in MWNTs to the oxygen atoms. (C) 2006 American Vacuum Society. [DOI: 10.1116/1.2180257]
\end{abstract}

\section{INTRODUCTION}

Carbon nanotubes (CNTs) constitute a class of onedimensional functional structures. Due to their structures and dependent electronic and mechanical properties, they have emerged as one of the foremost building blocks of nanoscale science. They are thought to have a number of potential applications, for example, as gas storage media, high strength composite materials, sensors, actuators, catalyst supports, field emission displays, nanoprobes, and as molecular wires for the next generation electronic devices. ${ }^{1-5}$ However, the application of as grown nanotubes is technologically difficult due to the nonreactive nature of the CNT surface, the presence of impurities (amorphous carbon, fullerenes, and catalyst particles), and the natural agglomeration of CNTs into bundles. To overcome these problems a modification of the carbon nanotubes by changing their surface chemical composition has proven to be efficient.

The use of oxidation reactions is often utilized as a purification method for cleaning raw CNT material and for chemical shortening of CNTs. ${ }^{6-12}$ For example, concentrated 3:1 $\mathrm{H}_{2} \mathrm{SO}_{4} / \mathrm{HNO}_{3}$ mixtures have been employed to cut highly entangled long ropes of CNTs into short open-ended pipes. As a result of the chemical oxidation, the ends and often the sidewalls of the nanotubes are covered with oxygen-containing groups such as carboxylate and ether groups. The carboxylic groups at the tube ends play an important role in CNT chemistry. They have been exploited for further derivatization and have served as a starting point for tethering a variety of different chemical moieties onto nano-

a) Author to whom correspondence should be addressed; electronic mail: p.papakonstantinou@ulster.ac.uk tube surfaces. In addition treatment in strong acids can also damage the walls of the nanotubes and thus produce carboneous impurities. The repulsive reactions between the covalent bonded oxygen groups prevent the aggregation into bundles.

Raman spectroscopy has been a sensitive probe of the electronic structure in carbon nanotubes and presence of defects. The $D$ mode at around $1300 \mathrm{~cm}^{-1}$ in the first order Raman spectra of CNTs is induced by disorder in a double resonance process. The relative intensity of this mode can provide direct evidence of covalent modification and defect concentration. When estimating the defect concentration, the $D$ mode intensity is usually normalized with respect to the intensity of the $G$ mode at around $1600 \mathrm{~cm}^{-1}$. This approach relies on the assumption that the intensity of the $G$ mode is independent of defect concentration and originates from a single resonant Raman process. On the other hand if the $G$ mode is defect induced, as was suggested in Ref. 13, this procedure for normalizing the intensity is no longer correct.

One of the main purposes of this article is to present first and second order Raman spectra of multiwalled carbon nanotubes (MWNTs) functionalized with oxygenated groups. The oxygen groups were introduced onto the nanotube surface through two strong acid purification routes: (1) reflux in concentrated (70\%) $\mathrm{HNO}_{3}$ acid for $4 \mathrm{~h}$ at $80{ }^{\circ} \mathrm{C}$ and (2) ultrasonification in $3 \mathrm{HNO}_{3}(70 \%): 1 \mathrm{H}_{2} \mathrm{SO}_{4}(98 \%)$ for $8.5 \mathrm{~h}$. By normalizing the intensity of the first order $D$ and $G$ peaks with respect to the second order overtone mode $D^{*}$, we show that not only the $D$ mode but also the $G$ mode is determined by a defect induced, double resonance process. In addition x-ray photoelectron spectroscopy (XPS) and thermal gravimetric analysis (TGA) have been used to obtain 


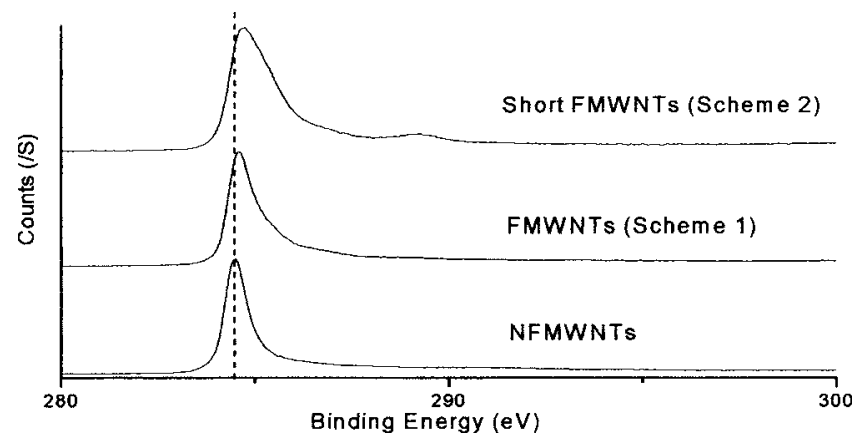

FIG. 1. XPS C1s peaks of NF, F (scheme 1), and S (scheme 2) nanotubes.

information on the chemical bonding configurations and purity of the MWNTs after the two strong acid treatments.

\section{EXPERIMENTAL PROCEDURES}

MWNTs made by thermal chemical vapor deposition (CVD) process were obtained after washing in $\mathrm{HCl}$ from Sun Nanotech Co. Ltd. People's Republic of China. They have average diameters of $10-30 \mathrm{~nm}$ and average lengths of $1-10 \mu \mathrm{m}$ and sample purity of $85 \%$. According to the manufacturer's specifications the $\mathrm{HCl}$ treated product was composed of $\sim 85 \%$ nanotubes, $\sim 10 \%$ amorphous carbon, and $\sim 5 \% \mathrm{NiO}$ and $\mathrm{FeO}$. These nanotubes are denoted as pristine (P). Subsequently, MWNTs were further purified under scheme 1. This involves refluxing the pristine nanotubes at concentrated $\mathrm{HNO}_{3}(70 \%)$ for $4 \mathrm{~h}$ at $80{ }^{\circ} \mathrm{C}$. The solid product was collected on a polytetrafluoroethylene (PTFE) membrane (Millipore porous filter, $0.2 \mu \mathrm{m}$ ) after diluting with de-ionized water until the filtrate $p \mathrm{H}$ became nearly neutral. The collected nanotubes on the membrane were dried overnight in an oven at $80{ }^{\circ} \mathrm{C}$. These nanotubes, purified under scheme 1 , are deemed as functionalized $(\mathrm{F})$.

MWNTs were also treated under scheme 2 . This involves sonication in a solution of $\mathrm{HNO}_{3}(70 \%) / \mathrm{H}_{2} \mathrm{SO}_{4}(98 \%)$ for $8.5 \mathrm{~h}$. The suspension was then diluted with water to neutral $p \mathrm{H}$ and filtered through a PTFE membrane under vacuum. After drying overnight the collected solid flaked off from the membrane. These tubes are referred to as short nanotubes (SMWNTs). The samples of nanotubes (pristine, FMWNTs, and SMWNTs) were suspended in organic solvent $N, N$-dimethylformamide (DMF) and sonicated for 2-3 h to allow even dispersion. Subsequently they were deposited onto a $\mathrm{Au}$ coated $\mathrm{Si}$ substrate using a drop dry technique producing a thick layer. Pristine nanotubes were nonsoluble in polar solvents such as DMF (were sunk at the bottom after several minutes), while the acid treated ones provided a black solution for several days which suggests that some hydrophilic groups such as carboxyl and hydroxyl are introduced and the tube ropes are unbundled to some extent. Pristine nanotubes sonicated in DMF are referred to as nonfunctionalized (NF).

Raman spectra were collected, employing a Labram 300 micro-Raman system. Two different lasers of wavelengths at $514.5 \mathrm{~nm}\left(\mathrm{Ar}^{+}\right.$ion) and $632.8 \mathrm{~nm}(\mathrm{He} / \mathrm{Ne})$ were used as

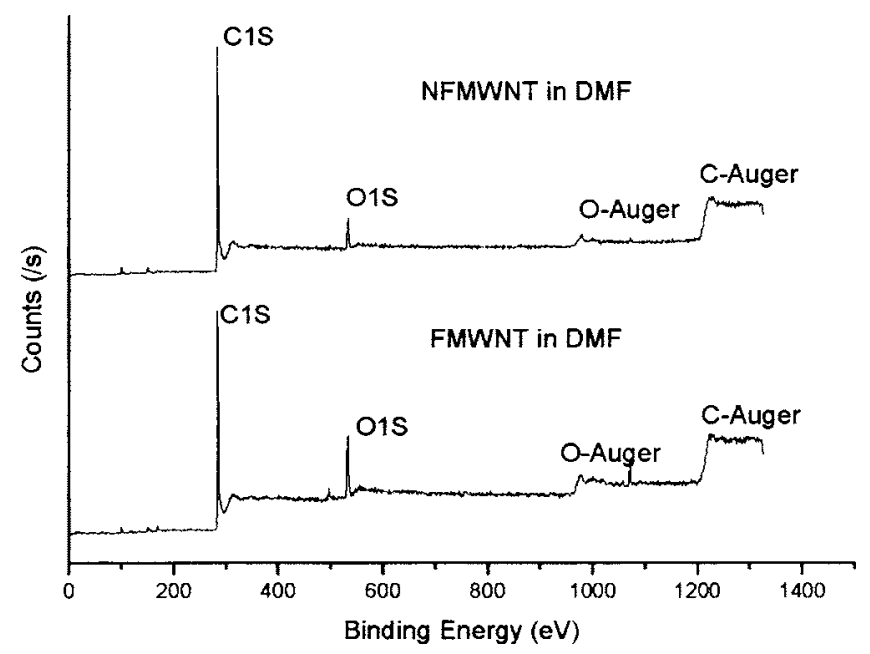

FIG. 2. XPS survey scan of NF and F nanotubes.

excitation sources. The micro-Raman system provides spectral resolutions of $1.4 \mathrm{~cm}^{-1}$ (for $514.5 \mathrm{~nm}$ ) and $1 \mathrm{~cm}^{-1}$ (for $632.8 \mathrm{~nm}$ ) per pixel with 1800 groove $/ \mathrm{mm}$ grating. The laser power impinging on the sample was kept constant at $3.5 \mathrm{~mW}$ with a $100 \times$ objective. Nine measurements were acquired at different locations on each sample. The Raman spectra were base line corrected using linear function and then the peaks were fitted using a Gaussian/Lorentzian function. Due to the high thickness of the deposited MWNT layers, surface enhanced Raman spectroscopy (SERS) related effects were not observed. XPS data were obtained using a high resolution electron spectroscopy for chemical analysis (ESCA) spectrometer. The TGA was done with Pyris 1 TGA analyzer (Perkin Elmer). The MWNT sample weight was $3.5 \mathrm{mg}$. The tests were performed under flowing nitrogen atmosphere at a temperature range between 90 and $860{ }^{\circ} \mathrm{C}$ with a step of $15^{\circ} \mathrm{C} / \mathrm{min}$.

\section{RESULTS AND DISCUSSION}

\section{A. XPS}

The treatment of MWNTs in acids leads to a drastic change in the position of the $\mathrm{C} 1 s$ core level. Figure 1 shows the $\mathrm{C} 1 s$ spectra of nonfunctionalized MWNT material (NF), after treatment under schemes $1(\mathrm{~F})$ and $2(\mathrm{~S})$. The core level of the samples $\mathrm{F}$ and $\mathrm{S}$ is shifted by $0.11-0.2$ and $0.46-0.55 \mathrm{eV}$ towards higher binding energies. Also their line shape is considerably broad and asymmetric at the high energy binding side. The degree of asymmetry increased drastically for the $\mathrm{S}$, giving $0.9,1.1$, and $1.4 \mathrm{eV}$ as the width at half maximum for $\mathrm{NF}, \mathrm{F}$, and $\mathrm{S}$ specimens, respectively. This suggests that there are several bonding configurations related to carbon based functional groups. Moreover an additional structure develops around $289 \mathrm{eV}$ binding energy for the $\mathrm{S}$ sample.

Figure 2 presents a full survey scan of NF and F. From these scans, the prominent peaks are the $\mathrm{C} 1 s$ and $\mathrm{O} 1 s$ and Auger peaks. A small amount of nitrogen $(\sim 1.5$ at. \%) is due to the immersion in DMF. ${ }^{14}$ Commonly metal catalysts 
TABLE I. Assigned percentage composition of carbon-containing surface groups for NF, F (scheme 1), and S (scheme 2) nanotubes.

\begin{tabular}{|c|c|c|c|c|c|c|c|c|}
\hline Sample & $\begin{array}{c}\mathrm{C}=\mathrm{C} \% \\
(284.5 \\
\mathrm{eV})\end{array}$ & $\begin{array}{c}\mathrm{C}-\mathrm{C} \% \\
(285.2 \\
\mathrm{eV})\end{array}$ & $\begin{array}{c}\mathrm{C}-\mathrm{O} \% \\
(286.25 \\
\mathrm{eV})\end{array}$ & $\begin{array}{c}\mathrm{C}=\mathrm{O} \% \\
(286.9 \\
\mathrm{eV})\end{array}$ & $\begin{array}{c}\mathrm{C}-\mathrm{OH} \\
\% \\
(287.8 \\
\mathrm{eV})\end{array}$ & $\begin{array}{c}\mathrm{HO}- \\
\mathrm{C}=\mathrm{O} \% \\
(288.9 \\
\mathrm{eV})\end{array}$ & $\begin{array}{c}\text { OCOO } \\
\% \\
(289.5 \\
\text { eV })\end{array}$ & $\begin{array}{c}\text { Satellite } \\
\text { peak } \\
(291 \\
\text { eV) }\end{array}$ \\
\hline $\mathrm{NF}$ & 55.22 & 14.61 & 8.37 & 4.91 & 3.39 & 3.55 & 3.81 & 6.14 \\
\hline F & 57.19 & 13.46 & 9.60 & 4.43 & 4.29 & 2.75 & 1.35 & 6.93 \\
\hline $\mathrm{S}$ & 32.71 & 34.74 & 12.15 & 5.71 & 6.47 & 5.01 & 1.59 & 1.63 \\
\hline
\end{tabular}

are located inside the nanotubes and/or are encapsulated by amorphous carbon or graphite layers. XPS is a surface sensitive technique with a probing depth between 1.5 and $6 \mathrm{~nm} .{ }^{15}$ Due to the limiting probe depth of XPS, Fe2P and $\mathrm{Ni} 2 P$ peaks originating from metal catalysts were not measurable.

The XPS analysis of $\mathrm{C} 1 s$ and $\mathrm{O} 1 s$ spectra of the NF, F, and $\mathrm{S}$ materials revealed that approximately $3.3 \%-5.5 \%$, $8 \%-14 \%$, and $16 \%$ of oxygen are bound to surface carbon atoms. The C1s envelope in the XPS spectrum of acid and nonacid treated nanotubes could be fitted using Gaussian function, summing peaks from eight types of carbon bonds: $\mathrm{C}=\mathrm{C}(284.5 \mathrm{eV}), \mathrm{C}-\mathrm{C}(285.2 \mathrm{eV}), \mathrm{C}-\mathrm{O}(286.25 \mathrm{eV})$, hydroxyls $\mathrm{C}-\mathrm{OH}(286.2 \mathrm{eV})$, carbonyls $\mathrm{C}=\mathrm{O}$ (aromatic: $286.9 \mathrm{eV}$ and aliphatic: $287.8 \mathrm{eV}$ ), carboxyls $\mathrm{HO}-\mathrm{C}=\mathrm{O}$ $(288.4-289.5 \mathrm{eV})$, carbonates OCOO $(289.5 \mathrm{eV})$, and a satellite peak $(291 \mathrm{eV})$. Table I provides the assigned percentage composition of carbon-containing surface groups for NF, $\mathrm{F}$, and $\mathrm{S}$ samples. These results provide evidence that surface oxygen groups were introduced to the carbon surface by acid treatment.

It has been generally accepted for oxidized graphites and also for carbon nanotubes that the carboxyl groups are mainly situated on the edges of the sheets and at the open ends of the tubes, whereas hydroxyl and carbonyl groups are most likely to be found on the basal planes and tube walls, respectively. The relatively higher concentration of $-\mathrm{COOH}$ in $\mathrm{S}$ nanotubes is consistent with the higher degree of open edges relative to that of $\mathrm{F}$ nanotubes.

The shift of the $\mathrm{C} 1 s$ line shape provides evidence for charge transfer between the oxygen molecule, present in the acids, and the carbon nanotubes. Oxygen and nitrogen have a higher electronegativity than carbon. Therefore nitric oxides can easily extract electrons from the tube wall shifting the $\mathrm{C} 1 s$ core level to the higher energy side. Similar shifting has been observed during fluorination of carbon nanotubes. ${ }^{16,17}$

\section{B. Raman spectroscopy}

Although Raman spectroscopy is a powerful tool to characterize single wall carbon nanotubes (SWNTs) it has not proven so successful with MWNTs. Most of the characteristic differences that distinguish between the Raman spectra in carbon nanotubes from that of graphite are not evident in MWNTs. The radial breathing modes (RBM), associated with large diameter tubes is too weak to be observed. The
Raman features associated with the small diameter inner tubes have been observed only under resonance conditions (SERS).

Figure 3 presents Raman spectra, acquired at $514.5 \mathrm{~nm}$ excitation, collected from (a) pristine (P) (nonfunctionalized material in powder form), (b) pristine material in thin film form (NF), (c) MWNTs functionalized under scheme $1(\mathrm{~F})$, and (d) MWNTs functionalized under scheme 2 (S). The most important features seen in Fig. 3 are the disorder induced $D$ band at $1320-1370 \mathrm{~cm}^{-1}$, its second harmonic $G^{\prime}$ (shoulder in $G$ ) at $1601-1612 \mathrm{~cm}^{-1}$, and the tangential $G$ band at $1530-1610 \mathrm{~cm}^{-1}$ which is related to the graphite tangential $E_{2 g}$ Raman active mode where the two atoms in graphene unit cell are vibrating tangentially one against the other. Second order weak bands also occur at 2682-2692 and $2914-3218 \mathrm{~cm}^{-1}$. Higher order Raman modes are also visible in the region of $4255-4320 \mathrm{~cm}^{-1}$. The spectra have been normalized to the highest peak intensity. Throughout the present article the intensity of the $D$ and $G$ band features refers to their most intense points, unless stated explicitly.

In Fig. 3, the RBMs observed in the low frequency region $\left(100-200 \mathrm{~cm}^{-1}\right)$ are broadened and their intensity is weak. This is related to the large diameter of the MWNTs under investigation. At a fixed $E_{\text {laser }}$ value the RBM linewidths are found to increase with increasing nanotube diameter. ${ }^{18}$

The $D$ band is activated in the first order scattering process of $s p^{2}$ carbons by the presence of in plane substitutional heteroatom vacancies, grain boundaries, or other defects and by finite size effects, all of which lower the crystalline symmetry of the quasiinfinite lattice. Therefore the $D$ mode can

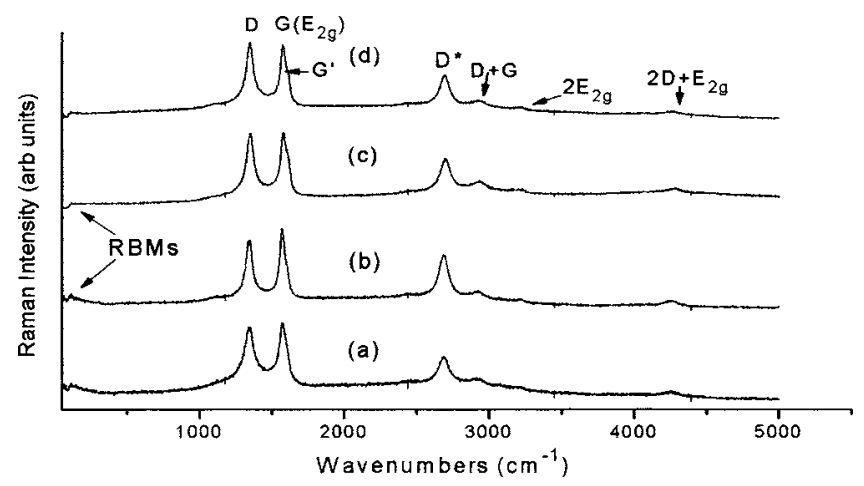

FIG. 3. Raman spectra at a laser of $514.5 \mathrm{~nm}$ excitation of P (powder) (a), NF (b), F (c), and S (d) nanotubes. 


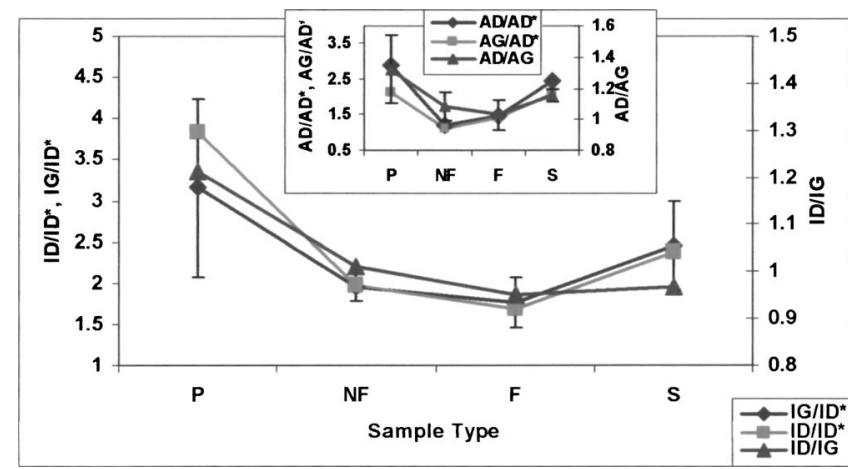

FIG. 4. Relative intensities: $I_{D} / I_{D^{*}}, I_{G} / I_{D^{*}}$, and $I_{D} / I_{G}$ of the Raman peaks for $\mathrm{P}, \mathrm{NF}, \mathrm{F}$, and $\mathrm{S}$ samples using a $514.5 \mathrm{~nm}$ excitation wavelength. Inset graph shows the corresponding integral area $(A)$ ratios: $A_{D} / A_{D^{*}}, A_{G} / A_{D^{*}}$, and $A_{D} / A_{G}$.

be used as a diagnostic of disruptions in the hexagonal framework of MWNTs and is induced by double resonance process. ${ }^{19}$

Usually the $I_{D} / I_{G}$ ratio is taken as a measure of defect concentration. It has been argued that the $G$ mode in the Raman spectra of carbon nanotubes originates from a defect induced double resonance scattering process. ${ }^{13}$ In order to get reliable information about the defect density it is necessary to include the intensity of the second order overtone mode $D^{*}$, which is due to two phonon processes and hence to first approximation independent of defect concentration.

To elucidate this controversy in Fig. 4 we show the relative intensities $\left(I_{D} / I_{D^{*}}, I_{G} / I_{D^{*}}\right.$, and $\left.I_{D} / I_{G}\right)$ of the Raman peaks as a function of treatment condition using a $514.5 \mathrm{~nm}$ excitation wavelength. The inset of Fig. 4 displays the corresponding ratios using the integrated area of the peaks. It is clear that both ratios based on intensity or area calculations show similar trends. A series of nine spectra was acquired at different spots placed approximately $1 \mathrm{~mm}$ apart on each sample and the ratios were averaged. Pristine CNTs in powder form possessed the largest deviations.

Very similar trends and magnitudes are observed for $I_{D} / I_{D^{*}}$ and $I_{G} / I_{D^{*}}\left(A_{D} / A_{D^{*}}\right.$ and $\left.A_{G} / A_{D^{*}}\right)$ ratios, which manifest that besides the $D$ mode, the $G$ mode in MWNTs originates from the same defect induced double resonance process. Therefore it is not appropriate to use the $G$ mode as basis for normalization. The ratio $I_{D} / I_{G}$ follows the same trend as well. However, the $I_{D} / I_{G}$ ratio varies only between 0.97 and $1.21\left(A_{D} / A_{G}\right.$ varies between 1.16 and 1.32).

Figure 5 presents the relative intensity and integrated area ratios for $632.8 \mathrm{~nm}$ excitation wavelength. Although very similar to those recorded at $514.5 \mathrm{~nm}$, the ratios have higher values upon excitation with longer excitation wavelength. However, all of them reveal a consistent trend. There is a reduction in $I_{D} / I_{D^{*}}$ (and $\left.A_{D} / A_{D^{*}}\right)$ upon sonification of pristine nanotubes in DMF or refluxing in nitric acid. In short MWNTs, $\mathrm{S}$ have a higher $I_{D} / I_{D^{*}}$ (and $A_{D} / A_{D^{*}}$ ) ratio compared to those treated in $\mathrm{HNO}_{3}$ or DMF.

The high $I_{D} / I_{D^{*}}$ (and $A_{D} / A_{D^{*}}$ ) ratio in $\mathrm{P}$ is related to the amorphous carbon originally present in the raw material. The

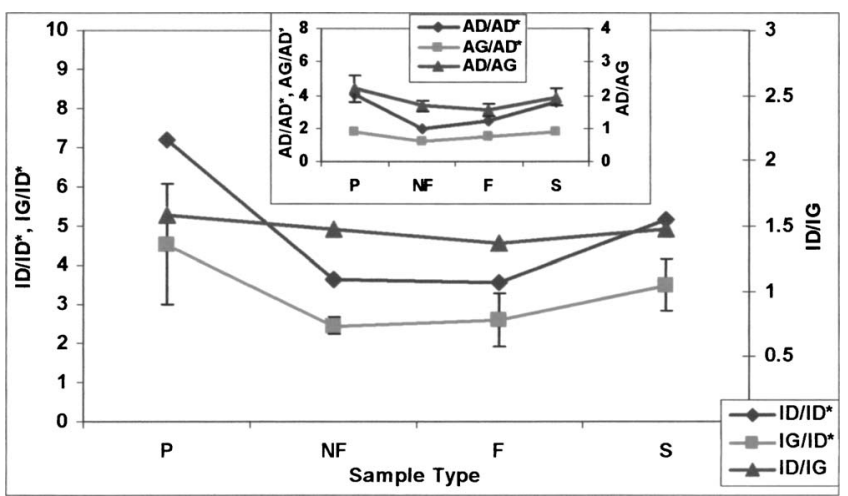

FIG. 5. Relative intensities: $I_{D} / I_{D^{*}}, I_{G} / I_{D^{*}}$, and $I_{D} / I_{G}$ of the Raman peaks for $\mathrm{P}, \mathrm{NF}, \mathrm{F}$, and $\mathrm{S}$ samples using a $632.8 \mathrm{~nm}$ excitation wavelength. Inset graph shows the corresponding integral area $(A)$ ratios: $A_{D} / A_{D^{*}}, A_{G} / A_{D^{*}}$, and $A_{D} / A_{G}$.

relative intensity of the $D$ mode decreases in $\mathrm{F}$, which indicates that reflux in nitric acid is an effective purification step for removing carbon impurities. Sonification in strong acids (scheme 2) produces significant destruction in MWNTs as evident from the higher relative intensity of $\mathrm{S}$ compared to that of $\mathrm{F}$. The increase in the relative intensity of the disorder mode $D$ in $\mathrm{S}$ can also be attributed to an increased number of $s p^{3}$ hybridized carbon in the nanotube framework, derived from the oxygen functionalization.

Many researchers have found that ultrasonication in an organic solvent leads to debundling or dispersion of carbon nanotubes. Treatment of the as grown nanotubes in $\mathrm{HCl}$ helps us to remove the metal nanoparticles and introduces strong hydrogen bonds which encourage bundling. Highly polar solvents such as DMF can disrupt the hydrogen bonding and disperse the nanotubes. Dispersion of SWNTs in organic amide solvents has been attributed to the availability of a free electron pair. ${ }^{20}$ Monthioux et al. found that sonication of their $3 M \mathrm{HNO}_{3}$ refluxed SWNT in DMF introduced extensive further damage to the tube walls. ${ }^{11}$

Figure 6 provides a comparison of the $D$ and $G$ peak positions for the $\mathrm{P}, \mathrm{NF}, \mathrm{F}$, and $\mathrm{S}$ nanotubes using the $514.5 \mathrm{~nm}$ excitation wavelength. In principle the position of the $D$ and $G$ lines is shifted to higher wave numbers

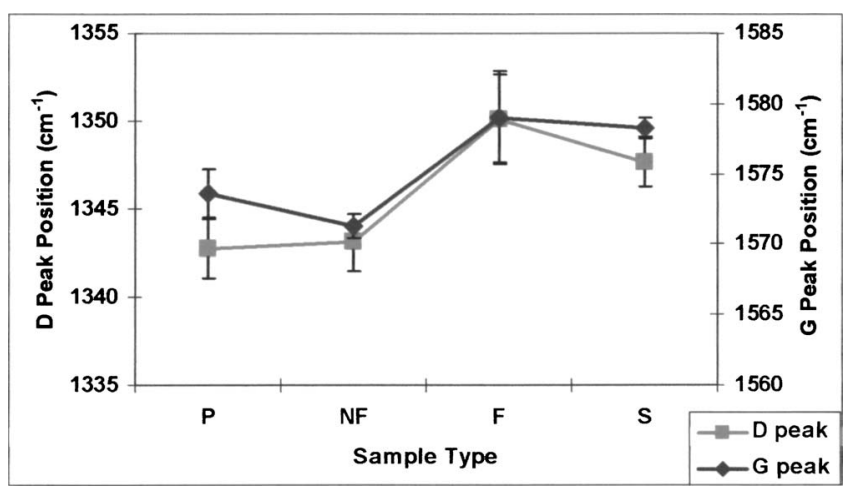

FIG. 6. Average $D$ and $G$ peak positions for P, NF, F, and $\mathrm{S}$ samples using a $514.5 \mathrm{~nm}$ excitation wavelength. 


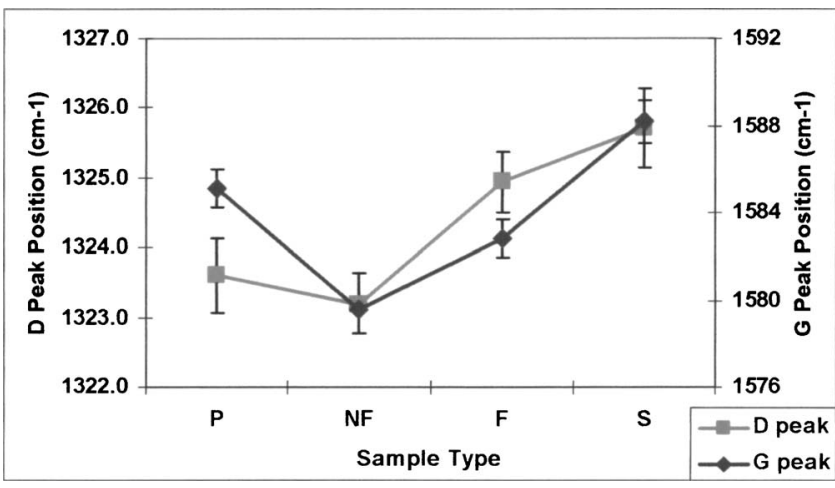

FIG. 7. Average $D$ and $G$ peak positions for $\mathrm{P}, \mathrm{NF}, \mathrm{F}$, and $\mathrm{S}$ samples using a $632.8 \mathrm{~nm}$ excitation wavelength.

$\left(\sim 7 \mathrm{~cm}^{-1}\right)$ for $\mathrm{F}$ and $\mathrm{S}$ nanotubes compared to $\mathrm{P}$ and $\mathrm{NF}$. Previous experimental and theoretical work has shown that the high frequency tangential modes of carbon atoms in SWNTs shift substantially to lower frequencies for electron donors ( $n$-type doping) or to higher frequencies for electron acceptors $^{21,22}$ ( $p$-type doping). Oxygen has an electronegative effect on the CNT structure acting like an electron withdrawing source similar to that of a $p$-type dopant. A plausible explanation for the observed upshift in the $G$ band would be electron transfer from the $\pi$ states in MWNTs to the oxygen atoms of nitric acid molecules, forming $\mathrm{NO}_{3}{ }^{-}$anions. The charge transfer behavior is in agreement with our XPS. This latter effect may be viewed as an intercalation of the nanotube lattice by oxidizing agents with the associated effects on the electronic properties of the nanotubes, a process which is quite familiar from the intercalation of graphite and fullerene agents by various acids. ${ }^{23}$ Indeed $\mathrm{HNO}_{3}$ intercalation has already been suggested by Bower et al. ${ }^{10}$ They identified an expansion in the inter-nanotube spacing and increase in the amount of hydrogen by $\mathrm{x}$-ray diffraction and proton NMR measurements after the SWNTs were immersed into the $\mathrm{HNO}_{3}$ for $2 \mathrm{~h}$.

The acid treatment leading to the intercalation of acid molecules inside the rope lattice will exert a pressure on the tubes. Such a pressure is likely to upshift the $D$ and $G$ positions. It has been observed that for carbon nanotubes under hydrostatic pressure the compression strain results in the shortening of $\mathrm{C}-\mathrm{C}$ bonds and corresponding shift of Raman $G$ peak to higher frequency. ${ }^{24}$ However, it is difficult to separate the effects of electron transfer from the carbon skeleton and the effects of strain due to the addition of acid molecules and anions in the interstitial channels between the tubes.

An upshift in $D$ and $G$ positions on the acid treated samples was also observed using the $632.8 \mathrm{~nm}$ excitation wavelength (Fig. 7). The position of the $D$ and $G$ lines is shifted to higher wave numbers $\left(\sim 3\right.$ and $\left.\sim 7 \mathrm{~cm}^{-1}\right)$ for $\mathrm{F}$ and $\mathrm{S}$ nanotubes compared to $\mathrm{P}$ and NF. The trends in $D$ and $G$ modes differ for 514.5 and $632.8 \mathrm{~nm}$ excitation wavelengths due to resonance effects. ${ }^{18}$ A comparison of Figs. 6 and 7 illustrates that the $D$ and $G$ bands are dispersive. The $D$ band frequency $\omega_{D}$ increases with laser excitation energy from 1323 to $1342 \mathrm{~cm}^{-1}$ (for the $\mathrm{P}$ nanotubes). The $G$ band fre-

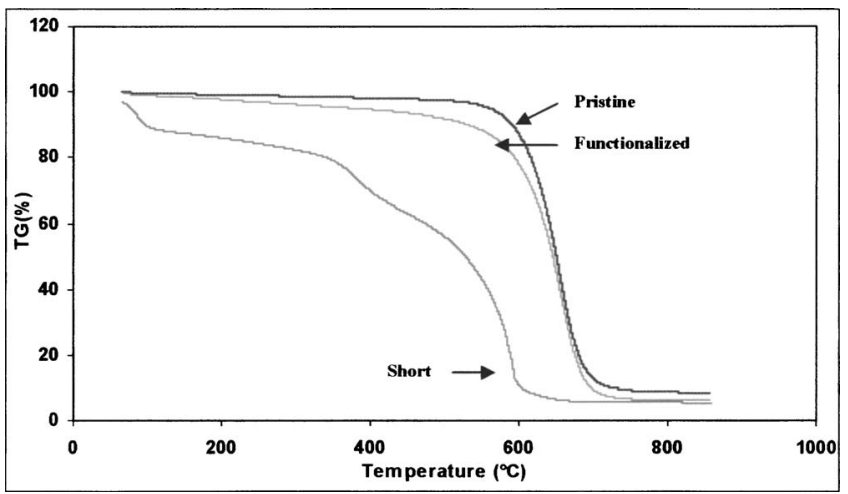

FIG. 8. TGA spectra of $\mathrm{P}, \mathrm{F}$, and $\mathrm{S}$ samples in flowing nitrogen atmosphere (residual weight: $\mathrm{P} \sim 10 \%, \mathrm{~F} \sim 6.3 \%$, and $\mathrm{S} \sim 4.3 \%$ ).

quency $\omega_{D}$ decreases to a smaller degree, with a laser excitation energy in the range of $1573-1585 \mathrm{~cm}^{-1}$ (for the $\mathrm{P}$ nanotubes). The excitation energy dependence provides a signature that the Raman $D$ and $G$ modes originate from double resonance effects.

\section{TGA}

TGA was used to determine the functionalization degree and the total amount of residual metals in the samples. Figures 8 and 9 show the TGA analyses of the P, F, and S samples. The weight loss derivative curves directly reflect the occurrence of thermal events (such as the onset of burning) as a function of temperature. It is obvious that the TGA and DTGA (derivative of thermogravimetry) curves of the $\mathrm{P}$ and $\mathrm{F}$ samples show similar shapes in the same weight loss temperature range. The appearance of higher slope in the TGA plot, at temperatures below $500{ }^{\circ} \mathrm{C}$ for sample $\mathrm{F}$, is associated with weight loss, caused by the detachment and fragmentation of oxygen group-terminated moieties. The residual components after thermal treatment at $850{ }^{\circ} \mathrm{C}$ are expected to be metal constituents. The residual weight fell from $10 \mathrm{wt} \%$ for the pristine material $\mathrm{P}$ to $6.3 \mathrm{wt} \%$ after acid treatment with scheme 1. DTGA plots of $\mathrm{P}$ and $\mathrm{F}$ samples show one single peak at about $650{ }^{\circ} \mathrm{C}$ associated with the decomposition of MWNTs.

In contrast, a two peak structure is observed in the DTGA curve of the $\mathrm{S}$ sample. The low temperature peak at around $380{ }^{\circ} \mathrm{C}$ is associated with amorphous carbon and oxygen

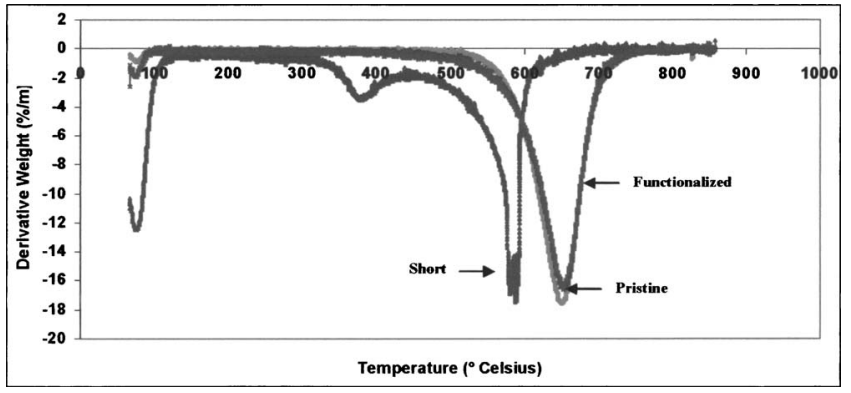

FIG. 9. Derivative TGA spectra of $\mathrm{P}, \mathrm{F}$, and $\mathrm{S}$ samples in flowing nitrogen atmosphere. 
groups. The sharp drop of the MWNT decomposition peak indicates that $\mathrm{S}$ nanotubes decompose at a much faster rate in comparison to $\mathrm{P}$ and $\mathrm{F}$ samples. Also the sharp weight loss at temperatures below $500{ }^{\circ} \mathrm{C}$, which corresponds to the removal of oxygen groups, offers a direct evidence for the different functionalization degree of scheme 2. The residual weight is reduced to 4.3 wt \%. As expected, scheme 2 resulted in a higher degree of functionalization (thus more weight loss), compared to that of scheme 1 . This observation is in agreement with the XPS data and Raman relative intensity ratios.

\section{SUMMARY}

We presented changes in the first and second order Raman spectra of multiwalled carbon nanotubes (MWNTs) induced by two acid treatment schemes. Scheme 1 involved refluxing of MWNTs in $\mathrm{HNO}_{3}$ acid and scheme 2 involved ultrasonification of $3: 1$ ratio in $\mathrm{HNO}_{3}$ and $\mathrm{H}_{2} \mathrm{SO}_{4}$. The intensity of both the $D$ and $G$ energy Raman modes if normalized to the second order mode $D^{*}$ mode follows similar trends upon acid treatments. Also the $D$ and $G$ peaks exhibited positive and negative shifts with increasing laser excitation energy. We interpret these results as an indication that the $G$ mode in carbon nanotubes is defect induced, in a similar manner to the $D$ peak. Both acid schemes cause an upshift of $D$ and $G$ Raman modes, due to intercalation of acid molecules exerting pressure on the $s p^{2}$ structure and extraction of electrons. XPS, TGA, and Raman intensity ratio results confirmed that scheme 2 produces a greater degree of functionalization and defect introduction.

\section{ACKNOWLEDGMENT}

This work was funded by the European Community under the FP6 DESYGN-IT grant. One of the authors, P.P. would like to thank Prof. Delichatsios for providing asscess to TGA apparatus.
${ }^{1}$ J. Wang, Electroanalysis 17, 7 (2005).

${ }^{2}$ M. Endo, T. Hayashi, Y. A. Kim, M. Terrones, and M. S. Dresselhaus, Philos. Trans. R. Soc. London, Ser. A 362, 2223 (2004).

${ }^{3}$ Y. Lin et al., J. Mater. Chem. 14, 527 (2004).

${ }^{4}$ W. I. Milne et al., J. Mater. Chem. 14, 933 (2004).

${ }^{5}$ R. H. Baughman, A. A. Zakhidov, and W. A. de Heer, Science 297, 787 (2002).

${ }^{6}$ K. J. Ziegler, Z. N. Gu, H. Q. Peng, E. L. Flor, R. H. Hauge, and R. E. Smalley, J. Am. Chem. Soc. 127, 1541 (2005).

${ }^{7}$ I. W. Chiang, B. E. Brinson, A. Y. Huang, P. A. Willis, M. J. Bronikowski, J. L. Margrave, R. E. Smalley, and R. H. Hauge, J. Phys. Chem. B 105, 8297 (2001).

${ }^{8}$ H. Hu, B. Zhao, M. E. Itkis, and R. C. Haddon, J. Phys. Chem. B 107, 13838 (2003).

${ }^{9}$ A. C. Dillon, T. Gennett, K. M. Jones, J. L. Alleman, P. A. Parilla, and M. J. Heben, Adv. Mater. (Weinheim, Ger.) 11, 1354 (1999).

${ }^{10}$ C. Bower, A. Kleinhammes, Y. Wu, and O. Zhou, Chem. Phys. Lett. 288, 481 (1998).

${ }^{11}$ M. Monthioux, B. W. Smith, B. Burteaux, A. Claye, J. E. Fischer, and D. E. Luzzi, Carbon 39, 1251 (2001)

${ }^{12}$ E. Borowiak-Palen et al., Chem. Phys. Lett. 363, 567 (2002).

${ }^{13}$ J. Maultzsch, S. Reich, and C. Thomsen, Appl. Phys. Lett. 81, 2647 (2002).

${ }^{14}$ T. I. T. Okpalugo, P. Papakonstantinou, H. Murphy, J. McLaughlin, and N. M. D. Brown, Carbon 43, 153 (2005).

${ }^{15}$ M. Stocker, Microporous Mater. 6, 235 (1996).

${ }^{16}$ Y. S. Lee, T. H. Cho, B. K. Lee, J. S. Rho, K. H. An, and Y. H. Lee, J. Fluorine Chem. 120, 99 (2003).

${ }^{17}$ P. R. Marcoux, J. Schreiber, B. Batail, S. Lefrant, J. Renouard, G. Jacob, D. Albertini, and J. Y. Mevellec, Chem. Phys. 4, 2278 (2002).

${ }^{18}$ M. S. Dresselhaus, G. Dresselhaus, A. Jorio, A. G. Souza Filho, and R. Saito, Carbon 40, 2043 (2002).

${ }^{19}$ C. Thomsen, S. Reich, and J. Maultzsch, Philos. Trans. R. Soc. London, Ser. A 362, 2337 (2004).

${ }^{20}$ C. A. Furtado, U. J. Kim, H. R. Gutierrez, L. Pan, E. C. Dickey, and P. C. Eklund, J. Am. Chem. Soc. 126, 6095 (2004).

${ }^{21}$ M. Rao, P. C. Eklund, S. Bandow, A. Thess, and R. E. Smalley, Nature (London) 388, 257 (1997).

${ }^{22}$ G. U. Sumanasekera, J. L. Allen, S. L. Fang, A. L. Loper, A. M. Rao, and P. C. Eklund, J. Phys. Chem. B 103, 4292 (1999).

${ }^{23}$ U. J. Kim, C. A. Furtado, X. Liu, G. Chen, and P. C. Eklund, J. Am. Chem. Soc. 127, 15437 (2005).

${ }^{24}$ P. M. Ajayan, L. S. Schadler, C. Giannaris, and A. Rubio, Adv. Mater. (Weinheim, Ger.) 12, 750 (2000). 